\title{
Cereal fibre intake and risk of mortality from all causes, CVD, cancer and inflammatory diseases: a systematic review and meta-analysis of prospective cohort studies
}

\author{
Maryam Hajishafiee ${ }^{1,2}$, Parvane Saneei ${ }^{1,2,3}$, Sanaz Benisi-Kohansal ${ }^{1,2}$ and Ahmad Esmaillzadeh ${ }^{4,5 *}$ \\ ${ }^{1}$ Food Security Research Center, Isfahan University of Medical Sciences, PO Box 81745-151, Isfahan, Iran \\ ${ }^{2}$ Department of Community Nutrition, School of Nutrition and Food Science, Isfahan University of Medical Sciences, PO Box \\ 81745-151, Isfahan, Iran \\ ${ }^{3}$ Students' Research Committee, Isfahan University of Medical Sciences, PO Box 81745-151, Isfahan, Iran \\ ${ }^{4}$ Department of Community Nutrition, School of Nutritional Sciences and Dietetics, Tehran University of Medical Sciences, PO \\ Box 14155/6117, Tehran, Iran \\ ${ }^{5}$ Diabetes Research Center, Endocrinology and Metabolism Clinical Sciences Institute, Tehran University of Medical Sciences, \\ PO Box 14155/6117, Tehran, Iran
}

(Submitted 4 November 2015 - Final revision received 10 April 2016 - Accepted 11 April 2016 - First published online 19 May 2016)

\section{Abstract}

Dietary fibre intake has been associated with a lower risk of mortality; however, findings on the association of different sources of dietary fibre with mortality are conflicting. We performed a systematic review and meta-analysis of the prospective cohort studies to assess the relation between cereal fibre intake and cause-specific mortality. Medline/PubMed, SCOPUS, EMBASE, ISI web of Science and Google scholar were searched up to April 2015. Eligible prospective cohort studies were included if they provided hazard ratios (HR) or relative risks (RR) and corresponding $95 \% \mathrm{CI}$ for the association of cereal fibre intake and mortality from all causes, CVD, cancer and inflammatory diseases. The study-specific HR were pooled by using the random-effects model. In total, fourteen prospective studies that examined the association of cereal fibre intake with mortality from all causes ( $n 48052$ death), CVD ( $n 16882$ death), cancer ( $n 19489$ death) and inflammatory diseases ( $n 1092$ death) were included. The pooled adjusted HR of all-cause mortality for the highest $v$. the lowest category of cereal fibre intake was $0.81(95 \%$ CI $0.79,0.83)$. Consumption of cereal fibre intake was associated with an $18 \%$ lower risk of CVD mortality (RR 0.82 ; $95 \%$ CI $0.78,0.86)$. Moreover, an inverse significant association was observed between cereal fibre intake and risk of death from cancer (RR 0.85; 95\% CI 0.81, 0.89). However, no significant association was seen between cereal fibre intake and inflammation-related mortality. This meta-analysis provides further evidence that cereal fibre intake was protectively associated with mortality from all causes, CVD and cancer.

Key words: Cereal fibres: Mortality: CVD: Cancer: Inflammation

Non-communicable diseases (NCD) are the main causes of death worldwide. CVD, cancers, chronic respiratory diseases and diabetes account for $60 \%$ of all deaths worldwide and $80 \%$ in low- or middle-income countries ${ }^{(1)}$. Several dietary factors including whole grains, fruits and vegetables have been shown to contribute to the risk for $\mathrm{NCD}^{(2-5)}$. Moreover, it seems that fibres from these dietary components have an important role in the aetiology of NCD and mortality ${ }^{(5-9)}$.

Earlier studies have shown that fibre intakes are inversely associated with all-cause mortality and CVD and cancer mortalities $^{(3,7,10,11)}$. A pooled analysis of ten prospective studies indicated that a $10 \mathrm{~g} / \mathrm{d}$ higher intake of total dietary fibre was associated with 17 and $27 \%$ reductions in the risk of all coronary events and coronary mortality, respectively ${ }^{(9)}$.
However, the results are inconsistent based on different food sources of dietary fibres ${ }^{(3-6,9)}$. It is suggested that greater intakes of cereal fibre are associated with a lower risk of CVD and all-cause mortalities ${ }^{(9,10,12)}$. Similar results have been reported by the National Institutes of Health (NIH)-AARP Diet and Health Study, which found that dietary fibre from grains, but not from other sources, was inversely related to cause-specific death in men and women ${ }^{(8)}$. In addition, in a cohort study on 3588 elderly, cereal fibre consumption was associated with a lower risk of CVD events ${ }^{(5)}$. However, some observational studies have not found a significant association between cereal fibre intake and CVD mortality ${ }^{(5,13)}$. Threapleton et al. $^{(14)}$ in UK Women's Cohort Study observed no significant association between cereal fibre intake and CVD mortality. Moreover, in

Abbreviations: HR, hazard ratio; MI, myocardial infarction; RR, relative risk.

* Corresponding author: A. Esmaillzadeh, fax +98 313668 1378, email esmaillzadeh@hlth.mui.ac.ir 
the cohort study of Women's Health Study among the US population, no significant association was observed between cereal fibre intake and CVD-specific death ${ }^{(4)}$. It is also suggested by the Blue Mountain Eye Study that cereal fibre intake is not associated with CHD or stroke mortality ${ }^{(15)}$. Two recent meta-analyses of cohort studies ${ }^{(16,17)}$ have reported the inverse associations of total dietary fibre intake with all-cause mortality; however, some relevant cohort studies were not included in these meta-analyses ${ }^{(3,10,12)}$. In addition, the relation between cereal fibre intake and cause-specific mortality was not examined in these meta-analyses. Nevertheless, in another meta-analysis by Liu et $a l .{ }^{(18)}$, which assessed the association of total dietary fibre intake with cause-specific mortality, different sources of dietary fibres were not considered. Given the lack of any clear meta-analysis of the association of cereal fibre intake with cause-specific mortality, as well as the conflicting evidence in this regard, we aimed to conduct a systematic review and meta-analysis of the prospective cohort studies to assess the relation between cereal fibre intake and mortality from all causes, CVD, cancer and inflammatory diseases.

\section{Methods}

\section{Search strategy}

A systematic literature search was conducted in medical databases including Medline/PubMed, SCOPUS, EMBASE, ISI web of Science and Google scholar up to April 2015 using the following medical subject headings (MeSH) and non-MeSH keywords relevant to 'whole grain', 'cereals' and 'dietary fiber' in combination with 'mortality', 'fatal', 'death' and 'survive'. No restriction was set on the time of publication and language. The reference lists of related articles were hand-searched for additional relevant studies. Titles/abstracts were screened for relevant studies by two independent investigators (M. H. and P. S.).

\section{Inclusion criteria}

Published studies were included if they were prospective cohort studies that reported relative risks (RR) or hazard ratio (HR) for the association of cereal fibre intakes and mortality from all causes, CVD, cancer and inflammatory diseases as the main outcomes of interest. In case of multiple published reports from the same study population, we included the one reporting the largest number of mortality cases ${ }^{(10,15)}$.

\section{Excluded studies}

We excluded editorial letters, comments, ecological studies and meta-analyses. The study selection process is illustrated in Fig. 1. Our search strategy identified 9895 human studies. After removing duplicates, 4798 abstracts were selected for a more detailed review; 181 citations were left after screening for title and abstracts. After scrutinising the full-text papers and based on our inclusion criteria, eighteen prospective studies were selected for the systematic review. However, we included only fourteen studies ${ }^{(3-5,9,10,12-14,15,19-23)}$ in the meta-analysis, and the other four studies were excluded from the meta-analysis because of the following reasons. Two studies conducted by Rimm et al. ${ }^{(24)}$ and Wolk et al. ${ }^{(25)}$ were not included because they had reported RR or HR for all CVD events simultaneously and had not separated the findings for fatal and non-fatal outcomes. Moreover, two studies were excluded because they had duplicated data from the same cohort studies ${ }^{(8,26)}$.

\section{Data extraction}

Two authors (M. H. and P. S.) extracted the required information independently. We used HR or RR as a measure of the association. First author's last name, publication year, cohort name, the country in which the study was conducted, sample size, age range of participants at baseline, methods of assessment of cereal fibre intake, duration of follow-up, main outcome, covariates adjusted for in the analyses and RR or HR with the corresponding 95\% CI were extracted. From each study, we extracted RR or HR estimates that reflected the greatest degree of adjustments for potential confounders. In case of disagreements between the two investigators, principal investigator (A. E.) was consulted.

\section{Quality assessment}

We assessed study quality with the Newcastle-Ottawa quality assessment scale ${ }^{(27)}$. This system allowed a total score of up to 9 points as the highest quality. Scores were derived through three aspects of each study including selection, comparability and outcome in eight questions. Each study could be awarded a maximum score of 1 for each question in selection and outcome categories. However, a maximum score of 2 could be given for comparability.

\section{Statistical methods}

$\mathrm{RR}$ and HR, and their $95 \% \mathrm{CI}$ for the comparison of the highest $v$. the lowest category of cereal fibre intake, were used to calculate $\log \mathrm{RR}$ and its standard error. The analyses were performed using random-effects model, calculating both $Q$-statistic and $I^{2}$ as indicators of heterogeneity. In case of significant between-study heterogeneity, we performed subgroup analysis, based on the location of the study, participants' sex, duration of follow-up and adjustment for energy intake, to find out possible sources of heterogeneity. Betweensubgroup heterogeneity was examined through fixed-effects modelling. We also performed random-effects meta-regression analysis to assess the overall linear relationship between cereal fibre intake and mortality. RR (95\% CI) comparing mortality from all causes, CVD, cancer and inflammatory diseases, in different categories of cereal fibre intake compared with the reference group, were extracted, converted to $\log \mathrm{RR}(\mathrm{SE})$ and used in this meta-regression. Depending on the available information in the included studies, the median or means of cereal fibre intake $(\mathrm{g} / \mathrm{d})$ in the categories were used. For studies in which a range of cereal fibre intake was reported, the mid-point of each category of intake was calculated. For categories with the open-ended ranges, half the width of the adjacent category was used. One study by Holmes et al. ${ }^{(21)}$ was not included in the meta-regression, because they had not reported the amount of cereal fibre intake across different 


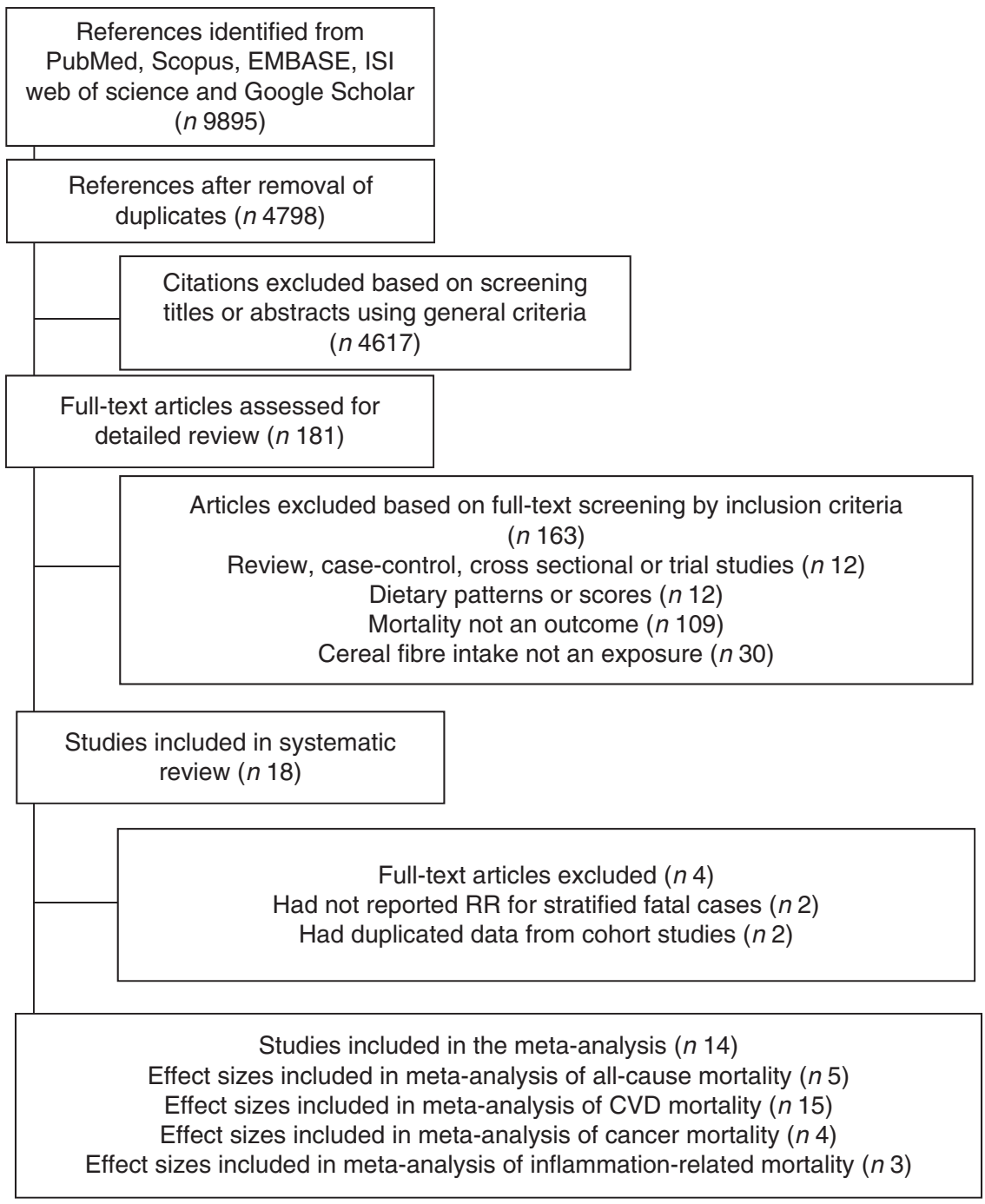

Fig. 1. The flow diagram of study selection. RR, relative risk.

categories. Moreover, the studies by Baer et al. ${ }^{(23)}$, Streppel et $a l^{(13)}$ and Pereira et $a l^{(9)}$, which had reported linear estimates (RR or HR for 4 or $10 \mathrm{~g} / \mathrm{d}$ increment in cereal fibre intake), were included in the meta-regression but not in the meta-analysis of the highest $v$. lowest intake. The publication bias was examined by visual inspection on Begg's funnel plots. Formal statistical assessment of funnel plot asymmetry was also carried out using Egger's regression asymmetry test. We also conducted a sensitivity analysis in which each prospective cohort study was excluded to examine the influence of that study on the overall estimate. Statistical analyses were conducted using Stata version 11.2 (StataCorp LP). $P$ values $<0.05$ were considered statistically significant for all tests including Cochran's $Q$ test.

\section{Results}

\section{Findings from the systematic review}

The characteristics of the studies included in the systematic review are presented in Table 1 . Out of eighteen cohort studies published between 1996 and 2015, ten were conducted in the $\mathrm{USA}^{(3-5,8,10,12,21,23-25)}$, two in the $\mathrm{UK}^{(14,19)}$, two in Australia ${ }^{(15,26)}$ and the rest in Japan ${ }^{(20)}$, Finland ${ }^{(22)}$ and Netherlands ${ }^{(13)}$, and one pooled analysis based on cohorts in the USA and $\mathrm{UK}^{(9)}$. The total number of participants in these studies ranged from $1373^{(13)}$ to $388122^{(8)}$, with age ranging from 30 to 99 years. Three ${ }^{(13,22,24)}$ studies were restricted to male subjects and six others to female subjects $(3,4,14,21,23,25)$. However, the remaining nine papers had examined both sexes, with three examining males and females separately ${ }^{(8,15,20)}$ and six in combination $^{(5,9,10,12,19,26)}$. From eighteen studies included in the systematic review, one study was conducted exclusively in patients with myocardial infarction (MI) ${ }^{(12)}$, and two studies were conducted in patients with type 2 diabetes and breast cancer, respectively ${ }^{(3,21)}$. From eighteen citations, six papers had reported RR for all-cause mortality $(3,8,10,12,13,23)$, seventeen publications for CVD mortality ${ }^{(3-5,8-10,12-15,19,20,22,23-26)}$, four studies for cancer mortality ${ }^{(8,10,21,23)}$ and three papers for inflammation-related mortality ${ }^{(8,10,15)}$. In some publications, cereal fibre was defined as fibre or NSP from flour, rice, pasta, 


\section{N British Journal of Nutrition}

Table 1. Main characteristics of prospective studies examined the association of cereal fibre intake with all-cause, CVD, cancer and inflammation-related mortalities (Mean values and standard errors; odds ratios (OR) or relative risk (RR) and $95 \%$ confidence intervals)

\begin{tabular}{|c|c|c|c|c|c|c|c|c|c|c|c|c|c|c|c|}
\hline $\begin{array}{l}\text { First author } \\
\text { (year) }\end{array}$ & Cohort name & Country & $\begin{array}{l}\text { Age range/ } \\
\text { mean age }\end{array}$ & Sex & Sample size & Cases & $\begin{array}{l}\text { Duration } \\
\text { of follow- } \\
\text { up (years) }\end{array}$ & Person-year & $\begin{array}{l}\text { Exposure and outcome } \\
\text { assessment }\end{array}$ & Outcome & Comparison & OR or RR & $95 \% \mathrm{Cl}$ & Score & Adjustments \\
\hline \multirow[t]{4}{*}{$\begin{array}{l}\text { Huang } \\
\qquad(2015)^{(10)}\end{array}$} & \multirow[t]{4}{*}{$\begin{array}{l}\text { NIH-AARP Diet } \\
\text { and Health } \\
\text { Study }\end{array}$} & \multirow[t]{4}{*}{ USA } & \multirow[t]{4}{*}{$50-71$} & \multirow[t]{4}{*}{$\mathrm{M} / \mathrm{F}$} & \multirow[t]{4}{*}{367442} & 46067 & \multirow[t]{4}{*}{14} & \multirow[t]{4}{*}{5148760} & \multirow[t]{4}{*}{$\begin{array}{l}\text { FFQ: } 124 \text {-item/NDI, Social } \\
\text { Security Administration } \\
\text { Death Master File, cancer } \\
\text { registry linkage }\end{array}$} & All-cause mortality & \multirow[t]{4}{*}{$\begin{array}{l}\text { Cereal fibre (ready to eat } \\
\text { cereals, high fibre cereals, } \\
\text { cooked cereals, grain-based } \\
\text { cereals) } \\
\text { Q5 v. Q1 }(10.22 \text { v. } 2.02 \mathrm{~g} / \mathrm{d})\end{array}$} & 0.81 & $0.79,0.84$ & \multirow[t]{4}{*}{8} & \multirow[t]{4}{*}{$\begin{array}{l}1,2,3,4,5,6 \\
\quad 7,8,9,11\end{array}$} \\
\hline & & & & & & 11283 & & & & CVD mortality & & 0.80 & $0.75,0.85$ & & \\
\hline & & & & & & 19043 & & & & $\begin{array}{l}\text { Cancer mortality } \\
\text { Infectious diseases- }\end{array}$ & & 0.85 & $0.81,0.89$ & & \\
\hline & & & & & & 922 & & & & $\begin{array}{l}\text { Infectious diseases- } \\
\text { mortality }\end{array}$ & & & & & \\
\hline \multirow[t]{2}{*}{$\mathrm{Li}(2014)^{(12)}$} & \multirow[t]{2}{*}{$\begin{array}{l}\text { NHS + HPFS } \\
\text { (patients with } \\
\text { MI) }\end{array}$} & \multirow[t]{2}{*}{ USA } & \multirow[t]{2}{*}{$30-75$} & \multirow[t]{2}{*}{$\mathrm{M} / \mathrm{F}$} & \multirow[t]{2}{*}{4098} & 1133 & \multirow[t]{2}{*}{$8 \cdot 7-9$} & \multirow[t]{2}{*}{37079} & \multirow[t]{2}{*}{$\begin{array}{l}\text { FFQ/vital records, NDI, family } \\
\text { reports, medical records or } \\
\text { autopsy }\end{array}$} & All-cause mortality & \multirow[t]{2}{*}{$\begin{array}{l}\text { Cereal fibre } \\
\text { Q5 v. Q1 (M: } 11 \cdot 2 \text { v. } 5 \cdot 3 ; \mathrm{F}: 8 \cdot 4 \\
\quad \text { v. } 4.0 \text { ) }\end{array}$} & 0.73 & $0.58,0.91$ & \multirow[t]{2}{*}{7} & $\begin{array}{l}1,2,3,4,5,6 \\
\quad 7,9,10,11\end{array}$ \\
\hline & & & & & & 558 & & & & CVD mortality & & 0.72 & $0.52,0.99$ & & \\
\hline $\begin{array}{l}\text { Threapleton } \\
\qquad(2013)^{(14)}\end{array}$ & $\begin{array}{l}\text { United Kingdom } \\
\text { Women's } \\
\text { Cohort Study }\end{array}$ & UK & $51 \cdot 8(\text { SE } 9 \cdot 2)^{\star}$ & $\mathrm{F}$ & 31036 & 258 & $14 \cdot 3$ & - & $\begin{array}{l}\text { FFQ: } 217 \text {-item/National death } \\
\text { registry }\end{array}$ & CVD mortality & $\begin{array}{l}\text { Cereal fibre (total cereal foods, } \\
\text { breakfast cereals) } \\
\text { Q5 v. Q1 }(15.7 \text { v. 2.8 g /d) }\end{array}$ & 0.91 & $0.56,1.48$ & 7 & $1,2,3,4,5,6,8$ \\
\hline $\begin{array}{l}\text { Crowe } \\
\qquad(2012)^{(19)}\end{array}$ & $\begin{array}{l}\text { European } \\
\text { Prospective } \\
\text { Investigation } \\
\text { into Cancer } \\
\text { and Nutrition- } \\
\text { Heart study }\end{array}$ & $\begin{array}{l}8 \text { European } \\
\text { countries }\end{array}$ & $53.8(\mathrm{SE} 8)^{\star}$ & $\mathrm{M} / \mathrm{F}$ & 306311 & 2381 & 11.5 & 3532887 & $\begin{array}{l}\mathrm{FFQ} / \text { Mortality registries at the } \\
\text { regional or national level }\end{array}$ & IHD mortality & $\begin{array}{l}\text { Cereal fibre (flour, pasta, rice, } \\
\text { other grains, bread, crisp } \\
\text { breads, rusks, breakfast } \\
\text { cereals, crackers, pastry, } \\
\text { pizza dough) g/d } \\
(\geq 11 \mathrm{v} .<5 \mathrm{~g} / \mathrm{d})\end{array}$ & 0.83 & $0.72,0.96$ & 8 & $\begin{array}{l}1,2,4,5,6 \\
\quad 7,8,9,10\end{array}$ \\
\hline \multirow[t]{3}{*}{$\begin{array}{l}\text { Baer } \\
\qquad(2011)^{(23)}\end{array}$} & \multirow[t]{3}{*}{ NHS } & \multirow[t]{3}{*}{ USA } & $52.5(\mathrm{sE} 7.1)^{\star}$ & $\mathrm{F}$ & 50112 & 4893 & 18 & - & $\begin{array}{l}\text { FFQ/family reports, NDI, } \\
\text { medical records }\end{array}$ & All-cause mortality & Cereal fibre (per 4g) & 0.84 & $0.78,0.91$ & 6 & $1,2,3,4,5,7,10$ \\
\hline & & & & & & $\begin{array}{r}1026 \\
931\end{array}$ & & & & $\begin{array}{l}\text { CVD mortality } \\
\text { Smoking-related } \\
\text { cancers-mortality }\end{array}$ & & $\begin{array}{l}0.82 \\
0.86\end{array}$ & $\begin{array}{l}0.69,0.97 \\
0.72,1.03\end{array}$ & & \\
\hline & & & & & & 1430 & & & & Other cancers- & & 0.82 & $0.71,0.95$ & & \\
\hline Park $(2011)^{(8)}$ & $\begin{array}{l}\text { NIH-AARP Diet } \\
\text { and Health } \\
\text { Study }\end{array}$ & USA & $50-71$ & M & 219123 & 20126 & 9 & - & $\begin{array}{l}\text { FFQ: } 124 \text {-item/NDI, Social } \\
\text { Security Administration } \\
\text { Death Master File, cancer } \\
\text { registry linkage }\end{array}$ & All-cause mortality & $\begin{array}{l}\text { Cereal fibre } \\
\text { Q5 v. Q1 }\end{array}$ & 0.77 & $0.73,0.81$ & 7 & $\begin{array}{c}1,2,3,4,5,6,7 \\
\quad 8,9,11\end{array}$ \\
\hline & & & & & & 5248 & & & & CVD mortality & & 0.77 & $0.71,0.85$ & & \\
\hline & & & & & & 8244 & & & & Cancer mortality & & 0.83 & $0.77,0.89$ & & \\
\hline & & & & & & 275 & & & & $\begin{array}{l}\text { Infectious diseases- } \\
\text { mortality }\end{array}$ & & 0.48 & $0.31,0.75$ & & \\
\hline & & & & $\mathrm{F}$ & 168999 & 11330 & & & & All-cause mortality & & 0.81 & $0.76,0.86$ & & \\
\hline & & & & & & 2417 & & & & CVD mortality & & 0.72 & $0.63,0.82$ & & \\
\hline & & & & & & $\begin{array}{r}4927 \\
223\end{array}$ & & & & $\begin{array}{l}\text { Cancer mortality } \\
\text { Infectious diseases- }\end{array}$ & & $\begin{array}{l}0.88 \\
0.89\end{array}$ & $0.80,0.97$ & & \\
\hline & & & & & & & & & & $\begin{array}{l}\text { minictious alseases- } \\
\text { mortality }\end{array}$ & & & & & \\
\hline $\begin{array}{l}\text { Buyken } \\
\qquad(2010)^{(15)}\end{array}$ & BMES & Australia & $>49$ & M & 1245 & 86 & 13 & - & $\begin{array}{l}\text { FFQ: } 145 \text {-item/Australian } \\
\text { National Death Index, family } \\
\text { reports }\end{array}$ & $\begin{array}{l}\text { Inflammatory } \\
\text { diseases-mortality }\end{array}$ & $\begin{array}{l}\text { Cereal fibre (breads and } \\
\text { cereals, pasta, rice) g/d } \\
\text { T3 v. T1 (M: } 11.5 \mathrm{v} .3 .0 \mathrm{~g} / \mathrm{d} \text {; } \\
\text { F: } 10.9 \mathrm{v} .2 .9 \mathrm{~g} / \mathrm{d} \text { ) }\end{array}$ & 0.98 & $0.57,1.67$ & 8 & $1,3,4,6,7,10$ \\
\hline & & & & $\mathrm{F}$ & 1490 & 84 & & & & & & 0.72 & $0.41,1.27$ & & \\
\hline & & & & M & 1245 & 151 & & & & CVD mortality & & 1.04 & $0.67,1.61$ & & \\
\hline & & & & $\mathrm{F}$ & 1490 & 109 & & & & & & 0.87 & $0.55,1.38$ & & \\
\hline $\begin{array}{l}\text { Eshak } \\
\qquad(2010)^{(20)}\end{array}$ & $\begin{array}{l}\text { The Japan } \\
\text { Collaborative } \\
\text { Cohort Study } \\
\text { for Evaluation } \\
\text { of Cancer } \\
\text { Risks }\end{array}$ & Japan & $40-79$ & M & 23119 & 231 & 14.3 & 288518 & FFQ: 40-item/death certificates & CHD mortality & $\begin{array}{l}\text { Cereal fibre (miso soup, rice) g/d } \\
\text { Q5 v. Q1 }(>2.1 \text { v. }<1.4 \mathrm{~g} / \mathrm{d})\end{array}$ & 0.89 & $0.65,1.01$ & 7 & $1,2,3,4,5,7,10$ \\
\hline & & & & $\mathrm{F}$ & 35611 & 191 & & 454653 & & & $(>1.7 \mathrm{v} .<1.1 \mathrm{~g} / \mathrm{d})$ & 0.76 & $0.59,0.97$ & & \\
\hline $\mathrm{He}(2010)^{(3)}$ & $\begin{array}{l}\text { NHS (type } 2 \\
\text { diabetic } \\
\text { patients) }\end{array}$ & USA & $30-55$ & $\mathrm{~F}$ & 7822 & 852 & 26 & 70102 & $\begin{array}{l}\text { FFQ/NDI, family reports, postal } \\
\text { system }\end{array}$ & All-cause mortality & $\begin{array}{l}\text { Cereal fibre (rice, pasta, bread, } \\
\text { breakfast cereals) } \\
\text { Q5 v. Q1 }(6.29 \text { v. } 1.9 \mathrm{~g} / \mathrm{d})\end{array}$ & 0.86 & $0.66,1.12$ & 7 & $\begin{array}{l}1,2,3,4,5,6,7 \\
10,11\end{array}$ \\
\hline & & & & & & 295 & & & & CVD mortality & & 0.89 & $0.57,1.41$ & & \\
\hline $\begin{array}{l}\text { Holmes } \\
\qquad(2009)^{(21)}\end{array}$ & $\begin{array}{l}\text { NHS (patients } \\
\text { with breast } \\
\text { cancer) }\end{array}$ & USA & $30-55$ & $\mathrm{~F}$ & 3846 & 446 & 25 & - & $\begin{array}{l}\text { FFQ/NDI, family reports, postal } \\
\text { system }\end{array}$ & $\begin{array}{l}\text { Breast cancer- } \\
\text { mortality }\end{array}$ & $\begin{array}{l}\text { Cereal fibre } \\
\text { Q5 v. Q1 }\end{array}$ & 1.00 & $0.71,1.40$ & 6 & $1,2,3,5,6,11$ \\
\hline
\end{tabular}




\section{Writish Journal of Nutrition}

Table 1. Continued

\begin{tabular}{|c|c|c|c|c|c|c|c|c|c|c|c|c|c|c|c|}
\hline $\begin{array}{l}\text { First author } \\
\text { (year) }\end{array}$ & Cohort name & Country & $\begin{array}{l}\text { Age range/ } \\
\text { mean age }\end{array}$ & Sex & Sample size & Cases & $\begin{array}{l}\text { Duration } \\
\text { of follow- } \\
\text { up (years) }\end{array}$ & Person-year & $\begin{array}{l}\text { Exposure and outcome } \\
\text { assessment }\end{array}$ & Outcome & Comparison & OR or RR & $95 \% \mathrm{Cl}$ & Score & Adjustments \\
\hline $\begin{array}{l}\text { Kaushik } \\
\qquad(2009)^{(26)}\end{array}$ & BMES & Australia & $\geq 49$ & $\mathrm{M} / \mathrm{F}$ & 2897 & 95 & 13 & - & $\begin{array}{l}\text { FFQ: } 145 \text {-item/Australian } \\
\text { National Death Index }\end{array}$ & Stroke mortality & $\begin{array}{l}\text { Cereal fibre } \\
\text { T1 v. T3 }\end{array}$ & 2.13 & $1 \cdot 19,3 \cdot 80$ & 7 & $1,2,3,6,8,10$ \\
\hline $\begin{array}{l}\text { Streppel } \\
\qquad(2008)^{(13)}\end{array}$ & $\begin{array}{l}\text { The Zutphen } \\
\text { Study }\end{array}$ & Netherlands & $80(\mathrm{SE} 3)^{\star}$ & M & 1373 & 1130 & 40 & - & $\begin{array}{l}\text { Dietary history/clinical } \\
\text { epidemiologist }\end{array}$ & All-cause mortality & $\begin{array}{l}\text { Cereal fibre (bread and other } \\
\text { cereal products) (per } 10 \mathrm{~g} / \mathrm{d} \\
\text { increment) }\end{array}$ & $\begin{array}{l}0.94 \\
\text { Recent intal } \\
\text { (95\% Cl } \\
\text { Long-ter } \\
0.86(95 \\
1.15) \\
\text { Recent intal } \\
\text { (95\% Cl } \\
\text { Long-ter } \\
0.90(95 \\
1.05)\end{array}$ & $\begin{array}{l}0.73,1.22 \\
\text { ke } 0.84 \\
\text { l } .64,1.10) \\
\text { m intake } \\
\% \text { Cl } 0.64, \\
\text { ke } 0.92 \\
0.79,1.07) \\
\text { m intake } \\
\% \text { Cl } 0.77\end{array}$ & 8 & $2,3,4,6,7,8$ \\
\hline $\begin{array}{l}\text { Pereira } \\
\qquad(2004)^{(9)}\end{array}$ & $\begin{array}{l}8 \text { prospective } \\
\text { cohort studies }\end{array}$ & $\begin{array}{l}\text { USA, } \\
\text { Europe }\end{array}$ & $35-98$ & M & 91058 & 2011 & $6-10$ & 2506581 & $\begin{array}{l}\text { FFQ or diet history/ } \\
\text { standardised criteria were } \\
\text { used for case ascertainment } \\
\text { in all studies }\end{array}$ & CVD mortality & $\begin{array}{l}\text { Cereal fibre (per } 10 \mathrm{~g} / \mathrm{d} \\
\text { increment) }\end{array}$ & 0.75 & $0.63,0.91$ & 6 & $\begin{array}{l}1,2,3,4,5,6,7 \\
\quad 8,10\end{array}$ \\
\hline $\begin{array}{r}\text { Mozaffarian } \\
(2003)^{(5)}\end{array}$ & $\begin{array}{l}\text { Cardiovascular } \\
\text { Health Study }\end{array}$ & USA & $\begin{array}{l}35-99 \\
\geq 65\end{array}$ & $\begin{array}{c}\mathrm{F} \\
\mathrm{M} / \mathrm{F}\end{array}$ & $\begin{array}{r}245186 \\
3588\end{array}$ & 220 & 8.6 & - & $\begin{array}{l}\text { FFQ: } 99 \text {-item/Medical records, } \\
\text { physician questionnaires, } \\
\text { death } \\
\text { certificates, medical } \\
\text { examiner forms, Health Care } \\
\text { Financing Administration } \\
\text { hospitalisations }\end{array}$ & IHD mortality & $\begin{array}{l}\text { Cereal fibre (dark breads, high } \\
\text { fibre or bran cereals) } \\
\text { Q5 v. Q1 }(>6.3 \text { v. }<1.7 \mathrm{~g} / \mathrm{d})\end{array}$ & 0.87 & $0.67,1.13$ & 6 & $1,3,4,5,7,8$ \\
\hline Liu (2002) $)^{(4)}$ & $\begin{array}{l}\text { Women's Health } \\
\text { Study }\end{array}$ & USA & $\geq 45$ & $\mathrm{~F}$ & 38480 & 570 & 6 & 230006 & $\begin{array}{l}\text { FFQ: } 131 \text {-item/medical records, } \\
\text { autopsy reports, death } \\
\text { certificates }\end{array}$ & CVD mortality & $\begin{array}{l}\text { Cereal fibre } \\
\text { Q5 v. Q1 (6.5 v. 3.0 g/d) }\end{array}$ & $1 \cdot 11$ & $0.84,1.46$ & 7 & $\begin{array}{l}1,2,3,4,5,6,7 \\
10,11\end{array}$ \\
\hline $\begin{array}{l}\text { Wolk } \\
\quad(1999)^{(25)}\end{array}$ & $\mathrm{NHS}$ & USA & $37-64$ & $\mathrm{~F}$ & 68782 & 591 & 10 & 641515 & $\begin{array}{l}\text { FFQ/NDI, family reports, postal } \\
\text { system }\end{array}$ & CHD-events & $\begin{array}{l}\text { Cereal fibre (median, g/d) } \\
\text { Q5 v. Q1 }\end{array}$ & 0.66 & $0.49,0.88$ & 7 & $\begin{array}{c}1,2,3,4,5,6,7 \\
10,11\end{array}$ \\
\hline $\begin{array}{l}\text { Pietinen } \\
\quad(1996)^{(22)}\end{array}$ & $\begin{array}{l}\text { Alpha-Tocopherol, } \\
\text { Beta-Carotene } \\
\text { cancer } \\
\text { prevention } \\
\text { study }\end{array}$ & Finland & $50-69$ & M & 21930 & 635 & 6.1 & 129388 & $\begin{array}{l}\text { FFQ: } 276 \text {-item/central } \\
\text { population register, death } \\
\text { certificates }\end{array}$ & Coronary mortality & $\begin{array}{l}\text { Cereal fibre } \\
\text { Q5 v. Q1 }(26.3 \text { v. } 8.8 \mathrm{~g} / \mathrm{d})\end{array}$ & 0.74 & $0.57,0.96$ & 6 & $\begin{array}{l}1,2,3,4,5,6,7 \\
\quad 8,10\end{array}$ \\
\hline$\underset{(1996)^{(24)}}{\operatorname{Rimm}}$ & HPFS & USA & $40-75$ & M & 43757 & 734 & 6 & - & $\begin{array}{l}\text { Dietary questionnaire: } 131 \text {-item/ } \\
\text { NDI, family reports, postal } \\
\text { system, medical records, } \\
\text { autopsy reports }\end{array}$ & $\begin{array}{l}\text { Total MI (fatal and non- } \\
\text { fatal) }\end{array}$ & $\begin{array}{l}\text { Cereal fibre } \\
\text { Q5 v. Q1 }(9.7 \text { v. } 2.2 \mathrm{~g} / \mathrm{d})\end{array}$ & 0.73 & $0.56,0.94$ & 6 & $\begin{array}{l}1,2,3,4,5,6,7 \\
10\end{array}$ \\
\hline
\end{tabular}

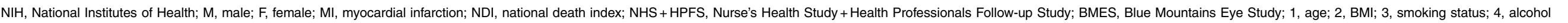

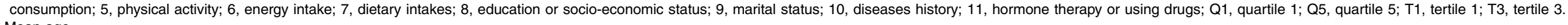

* Mean age. 
pastry, bread, dark bread and breakfast cereals ${ }^{(3,5,10,13,14,15,19,20)}$. However, others did not precisely report how they defined cereal fibre $^{(4,8,9,12,21,22,23-26)}$. Most studies had assessed dietary intakes by using a validated FFQ, whereas the remaining studies had used dietary questionnaire ${ }^{(24)}$ and diet history ${ }^{(13)}$. During the follow-up periods ranging from $6^{(4,24)}$ to 40 years ${ }^{(13)}$, there was a report of total number of 85531 death from all causes, 29352 from CVD, 35021 from cancer and 1590 inflammation-related deaths. Corresponding HR for all-cause mortality across the highest $v$. lowest categories of cereal fibre intake were between 0.73 and 0.92 . HR for CVD and cancer mortalities ranged from 0.66 to 2.13 and 0.82 to 1 , respectively. Moreover, reported HR for inflammation-related mortality ranged from 0.48 to 0.98 . Out of six citations with HR for all-cause mortality as the main outcome, four studies found a significant inverse association between cereal fibre intake and all-cause mortality in men and women $^{(8,10,12,23)}$. However, the remaining two studies did not show a significant association ${ }^{(3,13)}$. From seventeen publications that reported HR for CVD mortality, nine studies reported a significant inverse association between cereal fibre intake and CVD mortality ${ }^{(8-10,12,19,22-25)}$, whereas seven others did not $^{(3-5,13-15,26)}$. It is worth noting that one study found an inverse association between cereal fibre intake and CVD mortality in women but not in men ${ }^{(20)}$. Two out of four studies that examined the association of cereal fibre intake with cancer mortality reported a significant inverse association ${ }^{(8,10)}$. From three publications that reported HR for inflammation-related mortality, two studies did not find any significant association ${ }^{(10,15)}$, whereas one study reported a significant inverse association between cereal fibre intake and inflammation-related mortality in men but not in women ${ }^{(8)}$. Most studies had performed adjustments for important confounding factors including age ( $n$ 17), BMI ( $n$ 16), smoking ( $n$ 17) and alcohol consumption ( $n$ 16). Moreover, some studies had also made adjustments for physical activity ( $n$ 15) and energy intake ( $n$ 15) or other dietary variables or nutrients ( $n$ 15). In terms of the quality of the study, out of eighteen publications included in the systematic review six studies had a score $<7(0-6)$ and the rest had a score of $\geq 7$ (7-9).

\section{Findings from the meta-analysis on cereal fibre intake and all-cause mortality}

Three effect sizes from three publications ${ }^{(3,10,12)}$, including 379362 participants and 48052 cases of death, were included in this analysis (Fig. 2). Subjects with the highest intake of cereal fibre had $19 \%$ lower risk of all-cause mortality (RR 0.81; $95 \%$ CI $0.79,0.83)$, compared with those with the lowest intake. It should be taken into account that two studies from these three publications had been conducted on diabetic patients ${ }^{(3)}$ and individuals with $\mathrm{MI}^{(12)}$. No significant between-study heterogeneity was seen $\left(I^{2}=0 \cdot 0 \%, P=0 \cdot 60\right)$. Meta-regression of the five observational studies ${ }^{(3,10,12,13,23)}$ revealed that there is a significant inverse linear trend between cereal fibre intake $(\mathrm{g} / \mathrm{d})$ and risk of all-cause mortality $(\beta=-0 \cdot 019, P=0 \cdot 02)$ (Fig. 3(a)). Findings from the sensitivity analysis revealed that the exclusion of any single study from the analysis did not alter the overall association. Moreover, no evidence of publication bias was observed ( $P=0.80$ by Egger's test, $P=0.60$ by Begg's test).
Findings from the meta-analysis on cereal fibre intake and CVD mortality

Results from the meta-analysis on cereal fibre intake and CVD mortality are presented in Fig. 4. Overall, twelve effect sizes from ten studies ${ }^{(3-5,10,12,14,15,19,20,22)}$ were included. These studies had totally included 842172 participants at the time of study, out of which 16882 incident CVD deaths were reported. A significant inverse association was seen between highest consumption of cereal fibre and CVD mortality (RR 0.82; $95 \%$ CI 0.78, 0.86). After excluding two studies that had been performed on patients with $\mathrm{MI}^{(12)}$ and diabetes ${ }^{(3)}$, no significant changes occurred in the overall effect size (RR 0.82; 95\% CI 0.78, 0.86). Although no evidence of between-study heterogeneity was found $\left(I^{2}=0.0 \%\right.$, $P=0.62$ ), we performed a subgroup analysis based on sex, study location, follow-up duration and energy adjustment (Table 2). By sex, the inverse association tended to be stronger for men and women combined (RR 0.80; 95\% CI 0.76, 0.85) compared with women (RR 0.90; 95\% CI 0.77, 1.04) or men (RR 0.85; $95 \% \mathrm{CI}$ $0.72,1.00)$ separately. However, the difference was not statistically significant $(P=0 \cdot 38)$. According to study location, no significant difference was found between US and non-US countries $(P=0 \cdot 71)$. For the duration of follow-up, no statistical differences was seen between long-term $(\geq 10$ years) and short-term $(<10$ years) studies $(P=0.53)$. On the basis of energy adjustments, $R R$ for studies with energy adjustments tended to be stronger (RR $0.82 ; 95 \%$ CI $0.77,0.86)$ than studies without energy adjustments (RR 0.84; 95\% CI 0.73, 0.97). However, there was no statistically significant difference $(P=0 \cdot 68)$. As shown in Fig. 3(b), metaregression of the studies ${ }^{(3-5,9,10,12-15,19,20,22,23)}$ indicated that cereal fibre intake $(\mathrm{g} / \mathrm{d})$ was marginally associated with a lower risk of CVD mortality in a dose-response manner $(\beta=-0.007, P=0.06)$. No single study influenced the final association, and no evidence of publication bias was found ( $P=0.16$ by Egger's test, $P=0.34$ by Begg's test).

\section{Findings from the meta-analysis on cereal fibre intake and cancer mortality}

Results from two studies ${ }^{(10,21)}$, including 371288 participants and 19489 cancer death cases, revealed a significant inverse relationship between cereal fibre consumption and cancer mortality (RR 0.85; 95\% CI 0.81, 0.89). However, one of these studies had included patients with breast cancer ${ }^{(21)}$. No evidence of between-study heterogeneity was found $\left(I^{2}=0.0 \%\right.$, $P=0.35$ ). Findings from the meta-regression, using log RR of two studies ${ }^{(10,23)}$, demonstrated a marginally significant inverse relationship between cereal fibre intake $(\mathrm{g} / \mathrm{d})$ and risk of mortality from cancer $(\beta=-0 \cdot 016, P=0.09)$ (Fig. 3(c)). Excluding any single study did not affect this finding. In addition, no evidence of publication bias was seen ( $P=0.32$ by Begg's test).

\section{Findings from the meta-analysis on cereal fibre intake and inflammation-related mortality}

Results from the meta-analysis on cereal fibre consumption in relation to mortality from inflammatory diseases are presented in Fig. 5. No significant association was observed between 


\begin{tabular}{|c|c|c|c|c|}
\hline First author (year) & & $\mathrm{RR}$ & $95 \% \mathrm{Cl}$ & Weight (\%) \\
\hline $\mathrm{He}(2010)$ & & 0.86 & $0.66,1.12$ & 1.30 \\
\hline Li (2014) & & 0.73 & $0.58,0.91$ & $1 \cdot 80$ \\
\hline Huang (2015) & $\rightarrow$ & 0.81 & $0.79,0.84$ & $96 \cdot 90$ \\
\hline Overall $\left(I^{2}=0.0 \%, P=0.603\right)$ & & 0.81 & $0.79,0.83$ & $100 \cdot 00$ \\
\hline
\end{tabular}

Fig. 2. Forest plots of the association between cereal fibre intake and risk of all-cause mortality. RR, relative risk.

(a)

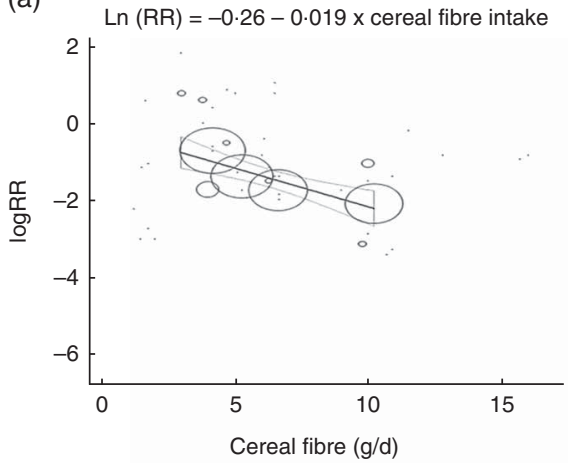

(c)

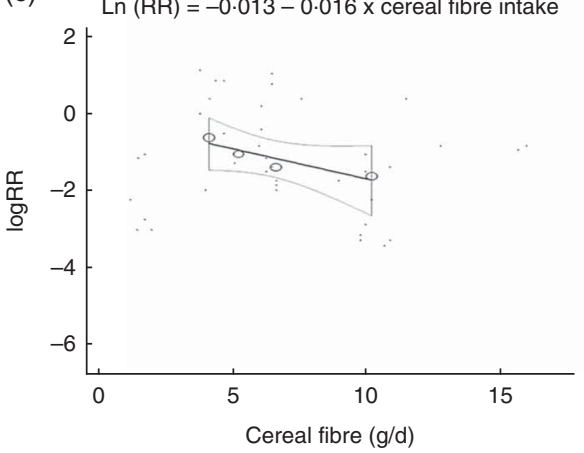

(b)

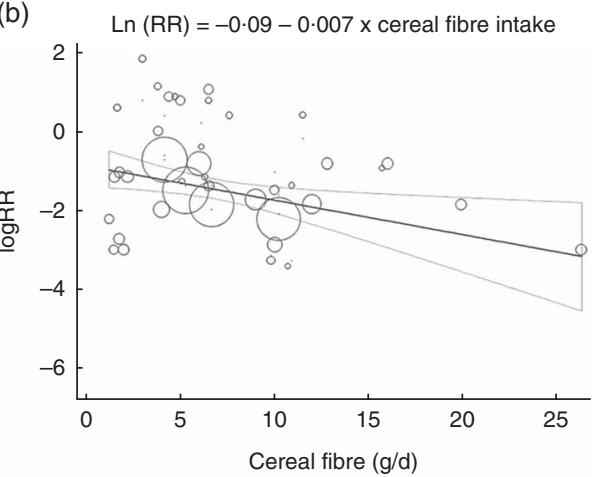

(d)

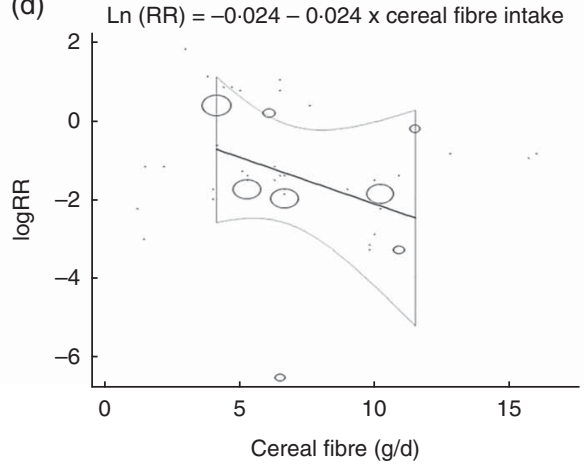

Fig. 3. (a) Association between the risk of all-cause mortality and cereal fibre intake: dose-response meta-regression. The levels of cereal fibre intake ( $\mathrm{g} / \mathrm{d})$ were modelled using a linear trend with random-effects meta-regression models. —_, Weighted regression line based on variance-weighted least squares. _, $95 \% \mathrm{Cl}$ around the regression line. $\bigcirc$, Relative risk (RR) in each study. The circle size is proportional to the precision of the RR. The vertical axis is on a log scale. For allcause mortality, $\beta=-0.019, P=0.02, I^{2}$ residual $=50.77 \%$. (b) Association between the risk of CVD mortality and cereal fibre intake: $\beta=-0.007, P=0.06$, $I^{2}$ residual $=0.00 \%$. (c) Association between the risk of cancer mortality and cereal fibre intake: $\beta=-0.016, P=0.09, I^{2}$ residual $=0.00 \%$. (d) Association between the risk of mortality from inflammatory diseases and cereal fibre intake: $\beta=-0.024, P=0.31, I^{2}$ residual $=3.86 \%$.

cereal fibre intake and inflammation-related mortality (RR 0.83; $95 \%$ CI $0.69,1 \cdot 01)$. Between-study heterogeneity was not statistically significant $\left(I^{2}=0.0 \%, P=0.73\right)$. We found that for the included studies ${ }^{(10,15)}$ there was no significant linear association between cereal fibre intake and inflammation-related mortality ( $\beta=-0 \cdot 024, P=0 \cdot 32$ ) (Fig. 3(d)). Moreover, excluding no single study affected the overall effect size. No evidence of publication bias was found ( $P=0.96$ by Egger's test, $P=0.60$ by Begg's test).

\section{Discussion}

We found that high cereal fibre intake was inversely associated with mortality from all causes, CVD and cancer. However, no significant association was found between cereal fibre intake and inflammation-related mortality. The inverse association with CVD mortality did not vary by sex, study location, followup duration and energy adjustment.

In the interpretation of our findings, it should be taken into account that in this meta-analysis the highest $v$. the lowest categories of cereal fibre intake have been used to demonstrate potentially important biological effects. We have also provided linear associations between cereal fibre intake and mortality. However, most nutritionists are wanting to know the appropriate amount of cereal fibre to recommend to the general population. As the range of intakes appeared to vary considerably between included studies, with the highest values in some studies being below the lowest values in other studies, current limited data did not allow us to determine a certain 


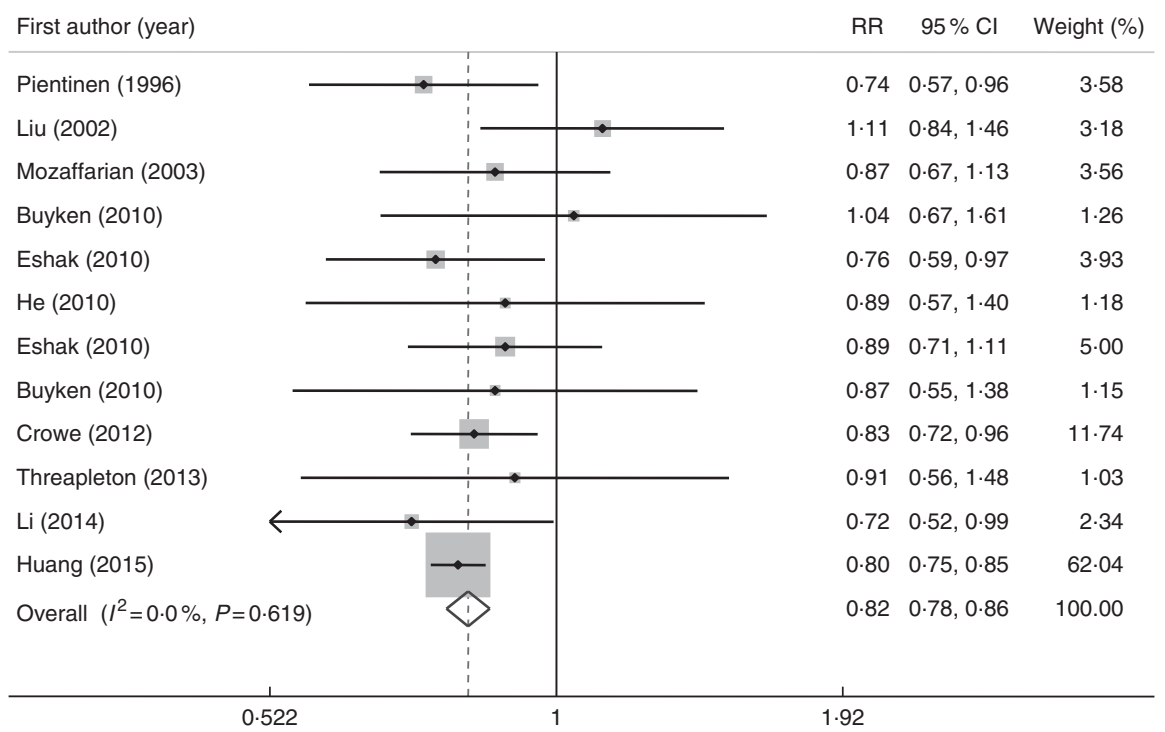

Fig. 4. Forest plots of the association between cereal fibre intake and risk of CVD mortality. RR, relative risk.

Table 2. Subgroup analysis for the association of cereal fibre intake and CVD mortality in prospective studies (Relative risks (RR) and $95 \%$ confidence intervals)

\begin{tabular}{|c|c|c|c|c|c|c|c|}
\hline & Effect sizes & No & $I^{2}$ & $Q$-test & $\mathrm{RR}$ & $95 \% \mathrm{Cl}$ & $P_{\text {Between }}$ \\
\hline \multicolumn{8}{|l|}{ Sex } \\
\hline Male $\mathrm{M}^{(15,20,22)}$ & 3 & 46294 & $3 \cdot 2$ & 0.35 & 0.85 & $0.72,1.00$ & \multirow[t]{3}{*}{0.38} \\
\hline Female ${ }^{(3,4,14,15,20)}$ & 5 & 114439 & 0.3 & 0.40 & 0.90 & $0.77,1.04$ & \\
\hline Both $(5,10,12,19)$ & 4 & 681439 & 0.0 & 0.79 & 0.80 & $0.76,0.85$ & \\
\hline \multicolumn{8}{|l|}{ Location } \\
\hline $\mathrm{USA}^{(3-5,10,12)}$ & 5 & 421430 & $34 \cdot 3$ & 0.19 & 0.85 & $0.75,0.96$ & \multirow[t]{2}{*}{0.71} \\
\hline Non-US ${ }^{(14,15,19,20,22)}$ & 7 & 420742 & 0.0 & 0.83 & 0.83 & $0.76,0.91$ & \\
\hline \multicolumn{8}{|l|}{ Duration } \\
\hline$<10$ years $^{(4,5,12,22)}$ & 4 & 68096 & $47 \cdot 5$ & 0.12 & 0.85 & $0.70,1.03$ & \multirow[t]{2}{*}{0.53} \\
\hline$\geq 10$ years ${ }^{(3,10,14,15,19,20)}$ & 8 & 774076 & 0.0 & 0.89 & 0.81 & $0.77,0.86$ & \\
\hline \multicolumn{8}{|l|}{ Energy adjustment } \\
\hline Yes $^{(3,4,10,12,14,15,19,22)}$ & 9 & 779854 & 0.0 & 0.44 & 0.82 & $0.77,0.86$ & \multirow[t]{2}{*}{0.68} \\
\hline $\mathrm{No}^{(5,20)}$ & 3 & 62318 & 0.0 & 0.62 & 0.84 & $0.73,0.97$ & \\
\hline
\end{tabular}

\begin{tabular}{|c|c|c|c|}
\hline First author (year) & RR & $95 \% \mathrm{Cl}$ & Weight (\%) \\
\hline Buyken (2010) & 0.98 & $0.57,1.68$ & $12 \cdot 27$ \\
\hline Buyken (2010) & 0.72 & $0.41,1.27$ & 11.09 \\
\hline Huang (2015) & 0.83 & $0.67,1.03$ & $76 \cdot 65$ \\
\hline Overall $\left(I^{2}=0.0 \%, P=0.738\right)$ & 0.83 & $0.69,1.01$ & $100 \cdot 00$ \\
\hline
\end{tabular}

Fig. 5. Forest plots of the association between cereal fibre intake and risk of mortality from inflammatory diseases. RR, relative risk.

value to recommend. It seems that further studies are required to shed light on this issue in the future.

Several observational studies have shown that dietary fibre intake, in particular cereal fibres, have protective effects against all-cause mortality, CVD events and different types of cancers $^{(3,5,12,20,21,24,25)}$. Findings of our meta-analysis were also in line with most previous publications. However, our findings about all-cause mortality, cancer mortality and inflammationrelated mortality should be interpreted with caution, because few studies were available to be included in the analysis. In addition, some studies had included subjects with diabetes and MI in their analysis ${ }^{(3,12)}$. Although the majority of studies that 
were included in our systematic review had reported a significant inverse association between cereal fibre intake and all-cause and CVD mortalities ${ }^{(8-10,12,19,22,23-25)}$, some studies did not reach a protective effect of cereal fibre intake on cancer and inflammation-related mortalities ${ }^{(10,15,21,23)}$. Such results were also seen in the NIH-AARP Diet and Health Study, in which cereal fibre intake was associated with a lower risk of total and cause-specific death in healthy men and women ${ }^{(8)}$. Findings from the Alpha-Tocopherol, Beta-Carotene Cancer Prevention study revealed that cereal fibre intake had stronger inverse association with coronary death than fibre from other sources in Finnish men ${ }^{(22)}$. Moreover, a study from the European Prospective Investigation into Cancer and Nutrition revealed a protective association between cereal fibre intake and CVD mortality. However, there was no significant difference in the association with outcome for fibre from different sources ${ }^{(19)}$. These findings are consistent with a recent meta-analysis in which cereal fibre intake, compared with other sources of dietary fibre, had the strongest inverse association with total mortality ${ }^{(16)}$. Mozaffarian et al. ${ }^{(5)}$ showed that cereal fibre intake was related to a lower risk of CVD events in the elderly, but reported no significant association between cereal fibre intake and CVD mortality. Other investigators have also failed to find a significant association between cereal fibre intake and CVD mortality $^{(4,15)}$. Similar results have also been reached in UK Women's Cohort study in which neither total dietary fibre nor cereal fibre intake was associated with CVD mortality ${ }^{(14)}$. In the Nurses' Health Study and Health Professionals Follow-up study with a large healthy population and long-term followup duration, an inverse association was seen between cereal fibre intake and mortality ${ }^{(23,24)}$. Moreover, pooled data of survivors of MI in these two large cohort studies revealed an inverse significant association between cereal fibre intake and all-cause and CVD mortalities ${ }^{(12)}$. Small study population, small number of events ${ }^{(5,15)}$ and short-term follow-up duration ${ }^{(5)}$ in some of these mentioned studies might explain the lack of a significant association between cereal fibre intake and CVD mortality. It must be noted that when FFQ was used to assess fibre intake in earlier studies an inverse association of dietary fibre intake with risk of mortality from all-causes, CVD and cancer $^{(4,5,14,15)}$ was found; however, this protective effect was not reached when diet history was applied for assessment of dietary fibre ${ }^{(13)}$. Moreover, different dietary fibre definitions among included studies could be problematic, and it might affect the estimates

Mechanisms by which cereal fibre intake might lower mortality from different causes are unknown. Cereals are one of the richest sources of insoluble fibres with laxative benefits ${ }^{(28)}$. Dietary fibres have particular effects on the composition of intestinal microbiota $^{(10)}$. Decreased faecal transit time and stool-bulking effect of insoluble fibre might also result in a lower contact of potential carcinogens to the colon ${ }^{(7,29,30)}$. Cereal fibre intake is a marker of other micronutrient and mineral-rich diet related to death caused by chronic diseases ${ }^{(2,5,7,12)}$. Diets high in cereal fibre are rich in folate, antioxidants, $\mathrm{Se}, \mathrm{Mg}, \mathrm{Cu}$, phenolic acids, lignans and phytochemicals $^{(31,32)}$, which might have a role in the protective association of cereal fibre with mortality. Cereal fibre might lead to lower levels of inflammatory markers including C-reactive protein and TNF- $\alpha$ receptor $2^{(10,15)}$. Lower levels of inflammation markers might explain, at least in part, the inverse association of cereal fibre intake with death. Moreover, it has been shown that high intake of cereal fibre was associated with a lower risk of type 2 diabetes ${ }^{(28)}$. High intake of cereals rich in insoluble fibres also has protective effects on postprandial blood glucose responses and insulin sensitivity ${ }^{(33)}$. The interference of cereal fibres with the absorption and digestion of dietary protein and the modulation of the metabolic signature of amino acids by inhibiting the activation of rapamycin/S6 kinase 1 signalling pathway are among the possible mechanisms ${ }^{(34,35)}$.

This study had several strengths. The first strength is the inclusion of prospective cohort studies that resulted in a large sample size with high statistical power. Although some studies had included patients as subjects in their analysis, we excluded them to reach findings generalisable to a healthy population. Most studies included in our meta-analysis had adjusted for confounding factors. However, the possibility of residual confounders cannot be excluded. Some limitations also need to be considered. Differences in sampling methods and dietary intake assessment tools might contribute to the variation in study findings. Because cereal fibre intake was assessed by FFQ in most included studies, measurement errors were inevitable. Moreover, different definitions used for categorisation of fibre consumption might result in mis-classifications. In addition, different definitions of cereal fibre among studies might also affect the associations. The possible relationship between cereal fibre consumption and healthier lifestyle might also attenuate true associations. Although we did not find publication bias, it is not easy to exclude such bias in any meta-analysis.

In conclusion, we found an inverse association between cereal fibre intake and mortality from all causes, CVD and cancer. No significant association was observed between cereal fibre and inflammation-related mortality.

\section{Acknowledgements}

This study was financially supported by Research Council of the Food Security Research Center, Isfahan University of Medical Sciences, Isfahan, Iran.

The financial support for conception, design, data analysis and manuscript drafting comes from Food Security Research Center, Isfahan University of Medical Sciences, Isfahan, Iran.

M. H. and P. S. contributed to conception, design, search, statistical analyses, data interpretation and manuscript drafting. S. B. K. contributed to design, search and data interpretation. A. E. contributed to conception, design, statistical analyses, data interpretation and manuscript drafting. A. E. supervised the study. All the authors approved the final manuscript for submission.

M. H., P. S., S. B. K. and A. E. declared no potential conflicts of interest.

\section{References}

1. Yach D, Hawkes C, Gould CL, et al. (2004) The global burden of chronic diseases: overcoming impediments to prevention and control. JAMA 291, 2616-2622. 
2. Erkkila AT, Herrington DM, Mozaffarian D, et al. (2005) Cereal fiber and whole-grain intake are associated with reduced progression of coronary-artery atherosclerosis in postmenopausal women with coronary artery disease. Am Heart J 150, 94-101.

3. He M, van Dam RM, Rimm E, et al. (2010) Whole-grain, cereal fiber, bran, and germ intake and the risks of all-cause and cardiovascular disease-specific mortality among women with type 2 diabetes mellitus. Circulation 121, 2162-2168.

4. Liu S, Buring JE, Sesso HD, et al. (2002) A prospective study of dietary fiber intake and risk of cardiovascular disease among women. J Am Coll Cardiol 39, 49-56.

5. Mozaffarian D, Kumanyika SK, Lemaitre RN, et al. (2003) Cereal, fruit, and vegetable fiber intake and the risk of cardiovascular disease in elderly individuals. JAMA 289, 1659-1666.

6. Buil-Cosiales P, Zazpe I, Toledo E, et al. (2014) Fiber intake and all-cause mortality in the Prevencion con Dieta Mediterranea (PREDIMED) study. Am J Clin Nutr 100, 1498-1507.

7. Larsson SC \& Wolk A (2014) Dietary fiber intake is inversely associated with stroke incidence in healthy Swedish adults. J Nutr 144, 1952-1955.

8. Park Y, Subar AF, Hollenbeck A, et al. (2011) Dietary fiber intake and mortality in the NIH-AARP diet and health study. Arch Intern Med 171, 1061-1068.

9. Pereira MA, O'Reilly E, Augustsson K, et al. (2004) Dietary fiber and risk of coronary heart disease: a pooled analysis of cohort studies. Arch Intern Med 164, 370-376.

10. Huang T, Xu M, Lee A, et al. (2015) Consumption of whole grains and cereal fiber and total and cause-specific mortality: prospective analysis of 367,442 individuals. BMC Med 13, 59 .

11. Krishnamurthy VM, Wei G, Baird BC, et al. (2012) High dietary fiber intake is associated with decreased inflammation and all-cause mortality in patients with chronic kidney disease. Kidney Int 81, 300-306.

12. Li S, Flint A, Pai JK, et al. (2014) Dietary fiber intake and mortality among survivors of myocardial infarction: prospective cohort study. BMJ 348, g2659.

13. Streppel MT, Ocké MC, Boshuizen HC, et al. (2008) Dietary fiber intake in relation to coronary heart disease and all-cause mortality over 40 y: the Zutphen Study. Am J Clin Nutr 88, 1119-1125.

14. Threapleton DE, Greenwood DC, Burley VJ, et al. (2013) Dietary fibre and cardiovascular disease mortality in the UK women's cohort study. Eur J Epidemiol 28, 335-346.

15. Buyken AE, Flood V, Empson M, et al. (2010) Carbohydrate nutrition and inflammatory disease mortality in older adults. Am J Clin Nutr 92, 634-643.

16. Kim Y \& Je Y (2014) Dietary fiber intake and total mortality: a meta-analysis of prospective cohort studies. Am J Epidemiol 180, 565-573.

17. Yang Y, Zhao LG, Wu QJ, et al. (2015) Association between dietary fiber and lower risk of all-cause mortality: a metaanalysis of cohort studies. Am J Epidemiol 181, 83-91.

18. Liu L, Wang S \& Liu J (2015) Fiber consumption and all-cause, cardiovascular, and cancer mortalities: a systematic review and meta-analysis of cohort studies. Mol Nutr Food Res 59, 139-146.

19. Crowe FL, Key TJ, Appleby PN, et al. (2012) Dietary fibre intake and ischaemic heart disease mortality: the European
Prospective Investigation into Cancer and NutritionHeart study. Eur J Clin Nutr 66, 950-956.

20. Eshak ES, Iso H, Date C, et al. (2010) Dietary fiber intake is associated with reduced risk of mortality from cardiovascular disease among Japanese men and women. J Nutr 140, $1445-1453$.

21. Holmes MD, Chen WY, Hankinson SE, et al. (2009) Physical activity's impact on the association of fat and fiber intake with survival after breast cancer. Am J Epidemiol 170, 1250-1256.

22. Pietinen P, Rimm EB, Korhonen P, et al. (1996) Intake of dietary fiber and risk of coronary heart disease in a cohort of Finnish men. The Alpha-Tocopherol, Beta-Carotene Cancer Prevention Study. Circulation 94, 2720-2727.

23. Baer HJ, Glynn RJ, Hu FB, et al. (2011) Risk factors for mortality in the nurses' health study: a competing risks analysis. Am J Epidemiol 173, 319-329.

24. Rimm EB, Ascherio A, Giovannucci E, et al. (1996) Vegetable, fruit, and cereal fiber intake and risk of coronary heart disease among men. JAMA 275, 447-451.

25. Wolk A, Manson JE, Stampfer MJ, et al. (1999) Long-term intake of dietary fiber and decreased risk of coronary heart disease among women. JAMA 281, 1998-2004.

26. Kaushik S, Wang JJ, Wong TY, et al. (2009) Glycemic index, retinal vascular caliber, and stroke mortality. Stroke $\mathbf{4 0}$, 206-212.

27. Wells G, Shea B, O'connell D, et al. (2000) The NewcastleOttawa Scale (NOS) for assessing the quality of nonrandomised studies in meta-analyses www.ohri.ca/programs/ clinical_epidemiology/oxford.htm (accessed April 2016).

28. Slavin J (2012) Fiber and prebiotics: mechanisms and health benefits. Nutrients 5, 1417-1435.

29. Stevenson L, Phillips F, O'Sullivan K, et al. (2012) Wheat bran: its composition and benefits to health, a European perspective. Int J Food Sci Nutr 63, 1001-1013.

30. Todd S, Woodward M, Tunstall-Pedoe H, et al. (1999) Dietary antioxidant vitamins and fiber in the etiology of cardiovascular disease and all-causes mortality: results from the Scottish Heart Health Study. Am J Epidemiol 150, 1073-1080.

31. Liu S, Stampfer MJ, Hu FB, et al. (1999) Whole-grain consumption and risk of coronary heart disease: results from the Nurses' Health Study. Am J Clin Nutr 70, 412-419.

32. McEligot AJ, Largent J, Ziogas A, et al. (2006) Dietary fat, fiber, vegetable, and micronutrients are associated with overall survival in postmenopausal women diagnosed with breast cancer. Nutr Cancer 55, 132-140.

33. Ames N, Blewett H, Storsley J, et al. (2015) A double-blind randomised controlled trial testing the effect of a barley product containing varying amounts and types of fibre on the postprandial glucose response of healthy volunteers. Br J Nutr 113, 1373-1383.

34. Hattersley JG, Pfeiffer AF, Roden M, et al. (2014) Modulation of amino acid metabolic signatures by supplemented isoenergetic diets differing in protein and cereal fiber content. J Clin Endocrinol Metab 99, E2599-E2609.

35. Weickert MO, Roden M, Isken F, et al. (2011) Effects of supplemented isoenergetic diets differing in cereal fiber and protein content on insulin sensitivity in overweight humans. Am J Clin Nutr 94, 459-471. 